\title{
Effect of procaine infiltration into biceps brachii muscle in man on supraspinal control of reflex transmission
}

\author{
PETER D. NEILSON ${ }^{1}$ \\ From the Division of Neurology, The Prince Henry Hospital, and \\ the Schools of Medicine and Physics, University of New South Wales, Sydney, Australia
}

SUMMARY Sensitivity of the tonic stretch reflex (TSR) of biceps brachii in normal man was measured at different levels of voluntary contraction before and after gamma efferent nerve fibres were selectively blocked by infiltration of dilute procaine. The procaine infiltration reduced TSR sensitivity to one half of its control value. It had no effect, however, on the slope of the relation between TSR gain and level of voluntary contraction. It was concluded that the increase in TSR sensitivity associated with voluntary contraction was not mediated by the gamma efferent fibres and control of reflex transmission is therefore not the main function of the fusimotor system.

The sensitivity of the tonic stretch reflex (TSR) of the biceps brachii muscle in man has been shown to increase with level of voluntary contraction, whereas in spastic patients the sensitivity is independent of contraction level (Neilson, 1972c). In spasticity the stretch reflexes impair the effectiveness of purposive voluntary movements and the loop-gain is set at a near maximal level so that reinforcement by voluntary contraction is unable to cause an increment in gain. Rushworth (1960) found that he was able to reduce muscle tone in gastrocnemius-soleus muscles in spastic patients, without reducing power, by injection of $1 \%$ procaine near the sciatic nerve. Based on evidence from previous studies (Matthews and Rushworth, 1958) in which procaine was shown to block differentially small diameter gamma efferent fibres, Rushworth concluded that an overactive fusimotor system contributes to muscle rigidity and spasticity. It has been shown that fusimotor activity can increase the slope of the relation between frequency of discharge of primary ending and the length of the muscle (Eldred, Granit, and Merton, 1953; Granit and Henatsch, 1956; Harvey and Matthews, 1961; Jansen and Matthews, 1962). Using differential nerve block

1 Centre Industries Research Scholar. in man by intrathecal and epidural procaine infusion, Landau, Weaver, and Hornbein (1960) confirmed the conclusion that procaine blocked the fusimotor fibres. The muscle spindle sensitivity to stretch was shown to be reduced, causing the tendon reflexes to disappear, even though the large diameter afferent and efferent fibres were largely unaffected. The aim of this study is to ascertain whether or not the increase in TSR sensitivity with contraction in normal subjects is caused by increased fusimotor activity associated with voluntary effort.

Using a cross-correlation technique of analysis it is possible to measure TSR transmission characteristics in normal subjects sustaining different levels of voluntary contraction (Neilson, 1972c). Repetition of the test before and after infiltration of a dilute procaine solution into the muscle belly should indicate whether or not the increase in TSR sensitivity in normal subjects is a consequence of increasing gamma efferent activity. Interpretation of the procaine block experiment relies on the conclusion that stretch reflex activity is depressed by procaine because it selectively blocks small gamma efferent fibres. This conclusion is the subject of some controversy. Gassel and Diamantopoulos (1964) point out considerable differences in various reports 
regarding the differential blocking of nerve fibres by procaine. From their own experiment they concluded that little or no differential blocking of gamma fibres occurs during procaine nerve block and that decrease in ankle-jerk and $\mathrm{H}$-reflex is in large part the direct result of a block of large afferent fibres. However, their observations concerned perineural procaine injection which is more difficult to control than the diffuse infiltration of a muscle belly which causes abolition of tendon reflexes with preservation of power and therefore seems acceptable as a form of differential block (Lance, 1970).

The role of fusimotor activity in the servomechanism theory of the control of movement (Merton, 1953) is not at all certain. As Matthews (1964) explained:

'In discussing the gamma route of excitation it is necessary to keep somewhat apart the evidence which indicates that the length of a muscle is maintained at a certain value by means of a servomechanism, and the evidence which suggests additionally that the null or balance point of the servo is normally changed by fusimotor activity acting alone. The evidence for the former idea is considerably stronger than that for the latter.'

Since fusimotor activity has been shown to increase the sensitivity of the primary spindle ending to stretch, it could be suggested that the main function of the fusimotor fibres is to control sensitivity of reflex transmission rather than set the intrafusal fibres to a desired length.

In the present study the subject attempted to keep the arm in a fixed position so that the desired length of the muscle should be constant. If fusimotor activity sets desired length by biasing the spindle, then it should also be constant during the test and could not, therefore, contribute to an increase in TSR sensitivity. On the other hand, if the main function of fusimotor activity is to control sensitivity of reflex transmission, then gamma activity would be expected to increase as the subject stiffens the arm and enhances TSR transmission. Thus this study is designed to assess the possible role of fusimotor activity in the servo model for control of movement.

\section{METHOD}

Five male subjects without a history of neurological disorder were tested before and after $10 \mathrm{ml}$. procaine
$1 \%$ was infiltrated into the biceps brachii muscle. The subject lay supine with the right arm strapped into a frame which constrained movement to flexion-extension about the elbow. Beckman surface $\frac{c}{0}$ EMG electrodes where attached $5 \mathrm{~cm}$ apart over the belly of the right biceps muscle to enable direct electromyogram (EMG) and integrated electromyogram (IEMG) to be recorded on an Offner four channel dynograph. Distortion introduced into the IEMG signal by the integrating filter was corrected $\overrightarrow{\vec{F}}$ by multiplying gain and phase curves by the inverse $\stackrel{?}{?}$ characteristics of the filter. The subject flexed the arm so that the elbow angle was in a $90^{\circ}$ position $\frac{\bar{c}}{\bar{c}}$ and was instructed to hold the arm as still as $\frac{\vec{\sigma}}{\overrightarrow{0}}$ possible in spite of disturbance forces applied to the $\varnothing$ limb by a spring connected $30 \mathrm{~cm}$ from the axis of $\%$ elbow rotation (Fig. 1). The other end of the spring $\overrightarrow{0}$ was connected to an oscillating cantilever arm on a sinusoidal stretching machine which has been $\vec{\omega}$ described elsewhere (Neilson, 1972b). Stretching the spring applied a sinusoidal disturbance torque about the elbow joint which was opposed by reflex con- $\dot{i}$ traction of the biceps muscle. The responses were ? involuntary, for frequencies greater than $2.0 \mathrm{~Hz}$ 迎 $\vec{\infty}$ least, since it has been shown that for these fre quencies the voluntary system is incapable of pro ducing coherent responses (Neilson, 1972a). Elbo $\overrightarrow{1}$ angle changes caused by the sinusoidal disturbane force were recorded on the dynograph by a gonior meter attached to the arm frame. Variations in ange were of the order of $10-20^{\circ}$ and were used as measure of change in muscle length. During eacti test the subject was instructed to stiffen the arm $\omega$ slowly by simultaneously contracting flexor and 0 extensor muscles. In this way the sensitivity of the TSR could be determined at different levels of voluntary contraction. Mean deflection of the IEMG signal above the baseline during each $1 \sec \frac{}{\varnothing}$ interval provided a measure of the level of voluntary $\underset{\Rightarrow}{\Rightarrow}$ contraction which was used to sort the data into five $\frac{0}{3}$ levels of contraction. The IEMG signal was calibrated by adjusting the pen deflection to $25 \mathrm{~mm}$ while the subject supported a $10 \mathrm{~kg}$ weight at the wrist. A cross-correlation analysis between the IEMG signal and elbow angle changes enabled the reflex responses? to be separated from the total electromyographic activity (Neilson, 1972b). Gain and phase of TSR pathways, taking elbow angle (muscle length) as input and IEMG responses as output, were measured at each level of voluntary contraction. These measurements were repeated before and after the $\rightarrow$ procaine infiltration. Since the duration of effective procaine block was limited to 5-10 min there was $N$ sufficient time only to measure gain and phase at 3 different frequencies.

Direct EMG was also displayed on a Tektronix 


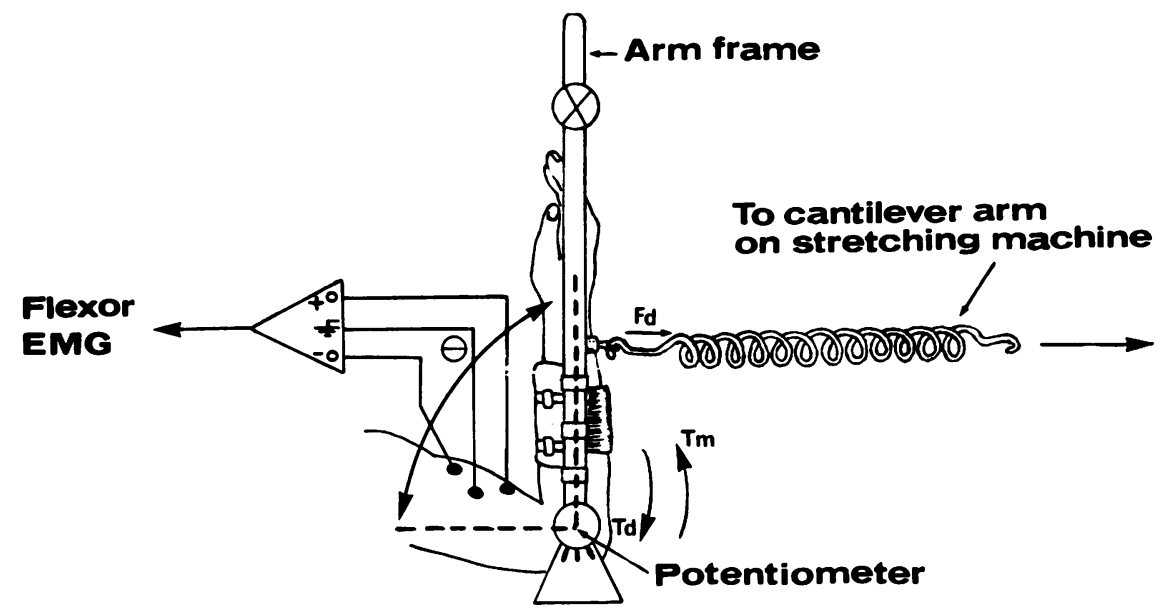

FIG. 1. Sketch of subject's arm strapped into arm frame. The subject was instructed to hold the arm as still as possible in the $90^{\circ}$ position in spite of disturbance forces applied through the spring.

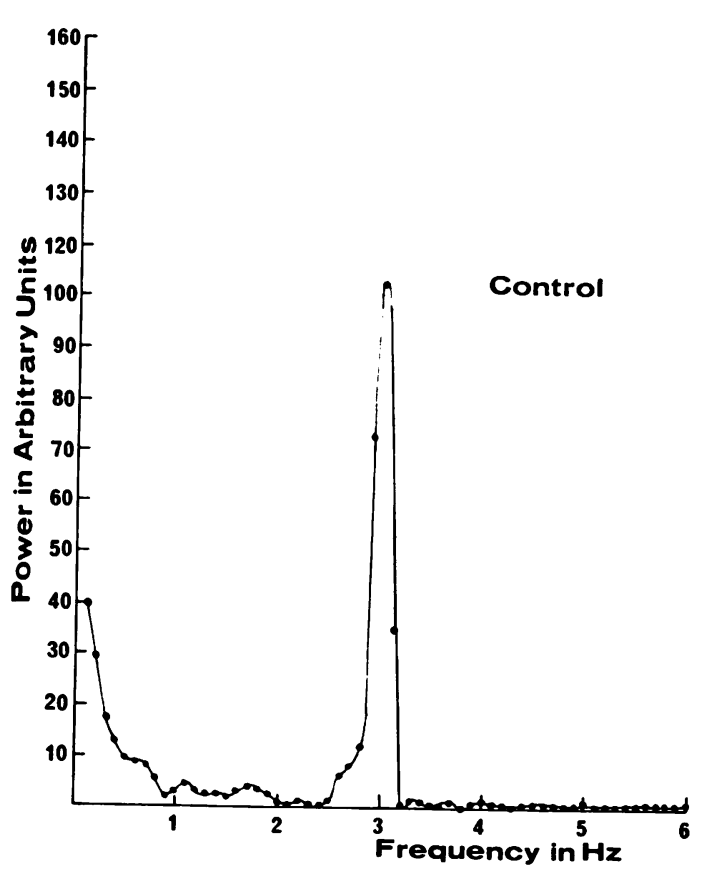

(a)

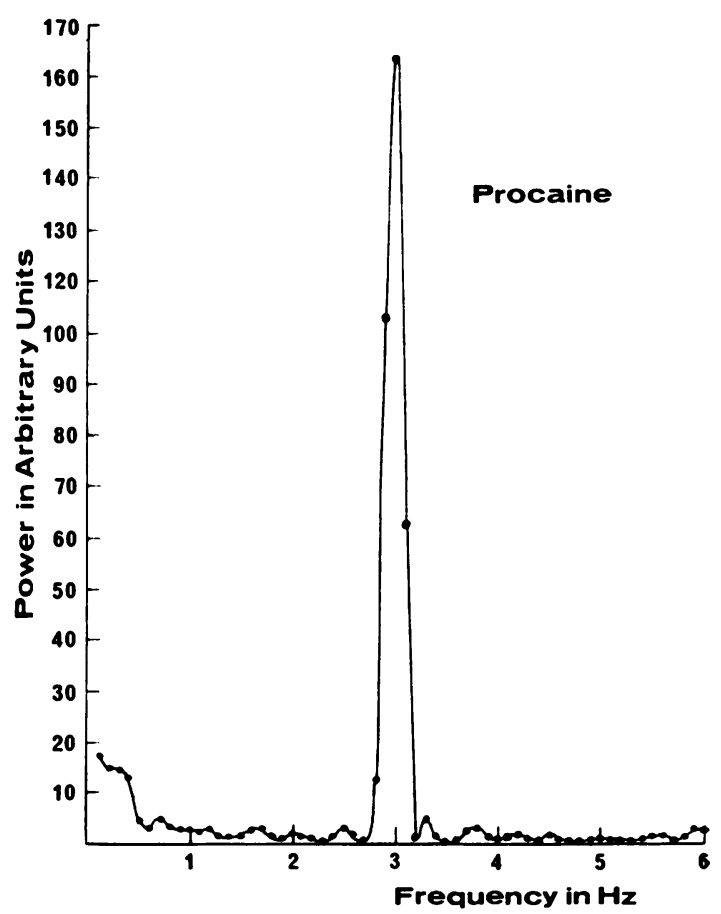

(b)

FIG. 2. Auto power spectra of elbow angle variation caused by a sinusoidal disturbance force $(3 \cdot 0 \mathrm{~Hz})$ (a) during a control test before procaine infiltration, and (b) after procaine was infiltrated into the biceps brachii muscle. The peak increased by about 33\% during the procaine block. 
531A oscilloscope after amplification in a Tektronix 122 preamplifier. This allowed a check for movement artefact and also enabled the synchronous EMG discharge of the biceps tendon jerk to be monitored. The effectiveness of the procaine block was assessed by the complete suppression of the EMG of the tendon-tap reflex responses without loss of muscle power. The tendon responses were tested regularly throughout the duration of the procaine block until the first sign of recovery of the response was observed. Testing was then terminated.

\section{RESULTS}

IEMG responses coherent with elbow angle changes were recorded before and after procaine infiltration of the biceps brachii muscle. At all levels of voluntary contraction the power spectra of elbow angle and IEMG signals displayed a peak at the stretching frequency (Figs 2 and 3). During the procaine block the peak in the elbow angle spectrum increased by $33 \%$ (Fig. 2a and Fig. 2b) indicating that the excursion of arm movement had increased and the peak in the IEMG spectrum decreased by $50 \%$ (Fig. 3a and Fig. 3b). These results show that the reflex responses were reduced to about half their pre- $\overline{\mathrm{D}}$ vious value in spite of an increase in both the magnitude and velocity of muscle stretching.

At every level of muscle contraction the coherence between IEMG and elbow angle signals at the disturbance force frequency de- $\frac{\hat{\Upsilon}}{\square}$ creased from a mean value of 0.8 to 0.6 after the administration of procaine. This drop in coherence shows that the IEMG signal contained $\vec{\Rightarrow}$ more fluctuations not correlated with elbow angle changes.

Phase lead of the IEMG signal ahead of $\frac{\overline{\bar{m}}}{\overline{2}}$ muscle stretch was calculated from the cross $\underset{\square}{\square}$ power spectrum. Procaine infiltration caused the phase lead to increase by a further $25^{\circ}-100^{\circ}$ 年 depending on contraction level. The total phase lead exceeded $180^{\circ}$ at the highest level of volun- $\vec{\omega}$ tary contraction, that is the maximum EMG occurred during the latter half of the shortening phase of the stretching cycle.

At all levels of voluntary contraction the procaine reduced TSR sensitivity to approximatefy one half its previous value, although the increase in gain with voluntary contraction was uñ affected (Fig. 4). The increase in phase lead of EMG ahead of muscle stretching described 음

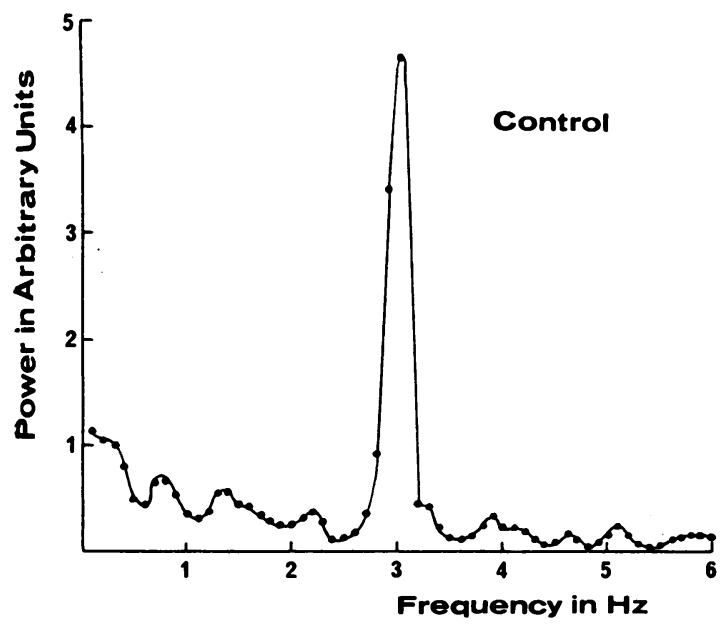

(a)

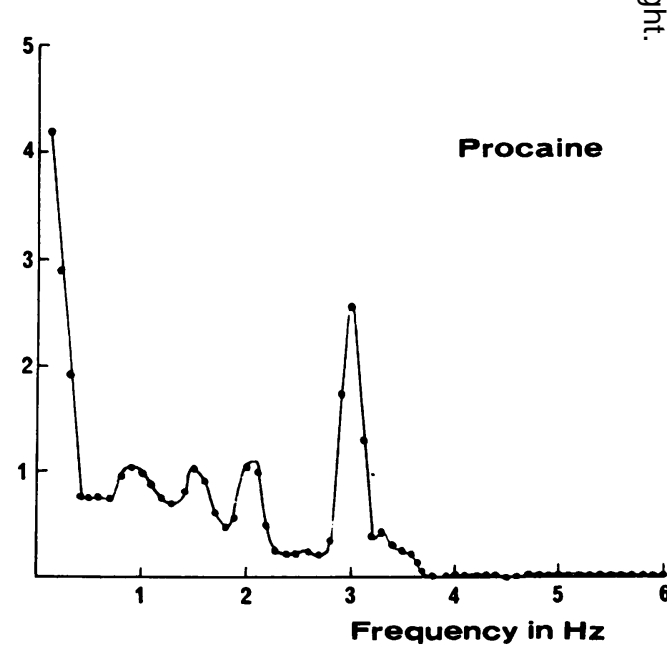

(b)

FIG. 3. Auto power spectra of variations in the IEMG signal recorded while a sinusoidal disturbance force $(3.0 \mathrm{~Hz})$ was applied to the limb (a) during a control test before procaine infiltration, and (b) after procaine was infiltrated into the biceps brachii muscle. The variation of IEMG at the disturbance frequency $(3.0 \mathrm{~Hz})$ decreased by $50 \%$ during the procaine block but there was an increase in power at lower frequencies. 


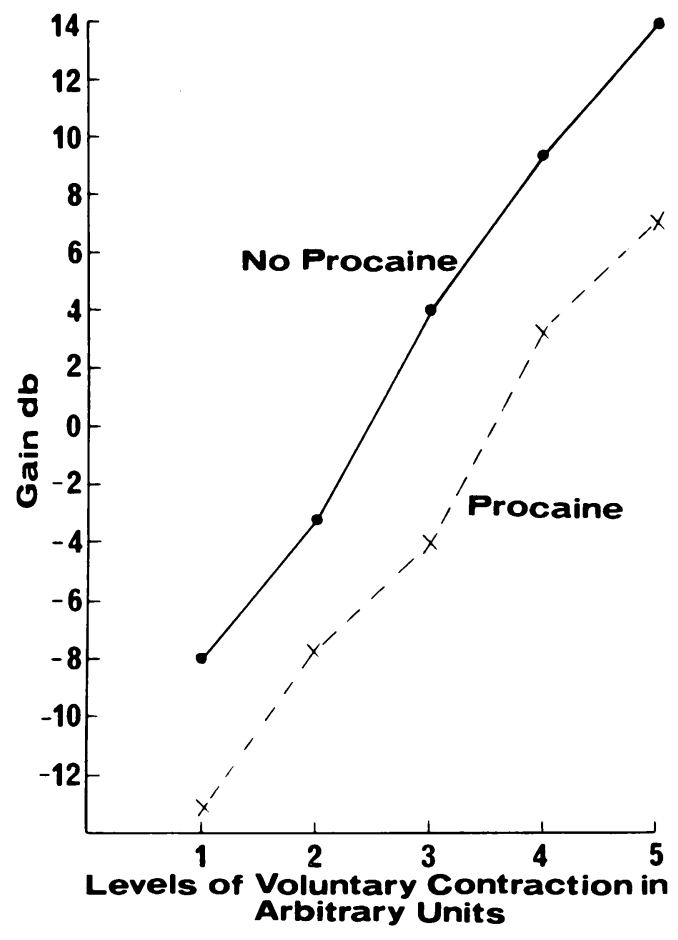

(a)

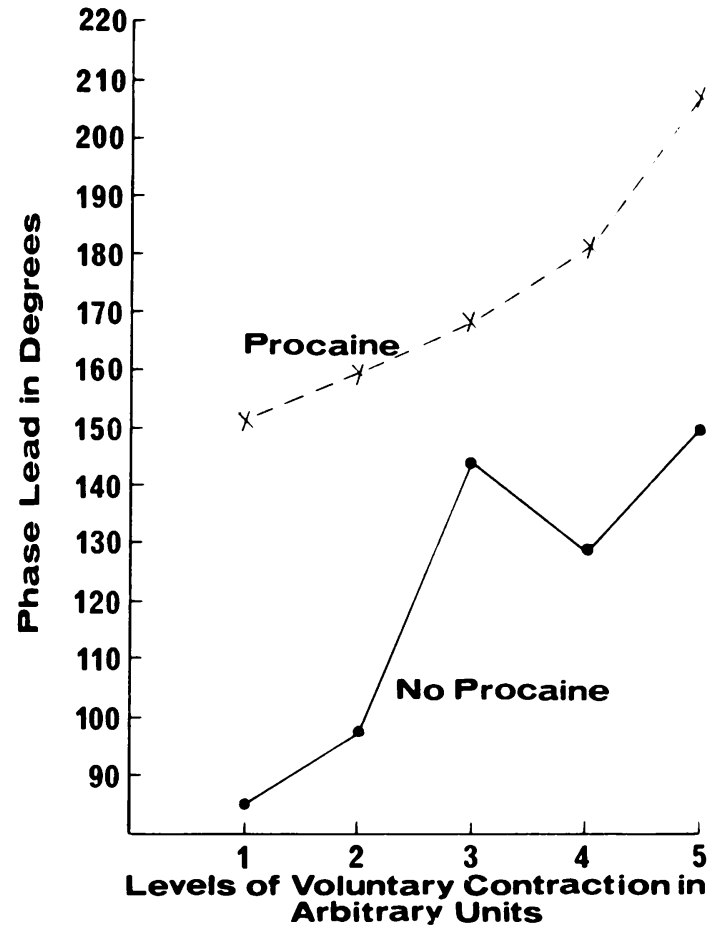

(b)

FIG. 4. (a) Gain of tonic stretch reflex pathways (ratio of change in IEMG to change in elbow angle) plotted against level of voluntary contraction, measured by the average IEMG deflection over 1 sec intervals. (1) during a control test before procaine infiltration, and (2) after procaine infiltration into the biceps muscle. Procaine reduced TSR gain by about $6 \mathrm{db}$ (one half) but did not influence the increase in gain with contraction. (b) Phase of TSR pathways plotted against level of voluntary contraction. Phase lead of EMG ahead of muscle stretch increased by $25^{\circ}-100^{\circ}$ during the procaine block and the phase lead continued to increase with voluntary contraction.

previously (Neilson, 1972c) was unchanged by the procaine infusion (Fig. 4).

\section{DISCUSSION}

Procaine infiltration into the biceps muscle completely blocked the biceps tendon jerk without reducing muscle power for a period of 5-10 $\mathrm{min}$ and thus appears to have been effective in producing a relatively selective block of the small diameter gamma efferent nerve fibres. The $50 \%$ reduction in the EMG of the TSR responses is consistent with the results reported by Rushworth (1961) in patients with Parkinsonism and spasticity and with the study reported by DeGail, Lance, and Neilson (1966) in which procaine infiltration into the quadriceps muscle in normal man reduced the tonic vibration reflex (TVR) to one third or less of its control value.

The increase in the size of the peak in the elbow angle power spectrum after the procaine block indicates that the amount of movement about the elbow at the stretching frequency was increased. The reflexes were less effective in compensating for disturbance forces applied to the limb and the subject's ability to hold the arm steady was diminished. The decrease in the size of the peak in the IEMG power spectrum indicates that procaine reduced the magnitude of the reflex response to about one half in spite of the increase in amplitude and velocity of stretching. The IEMG spectrum also showed an 
increase in the variance of the IEMG signal at frequencies other than the stimulus frequency after procaine infiltration. The IEMG of the TSR responses is therefore more difficult to measure after a procaine block because it is not only smaller but is mixed with a larger amount of variation of EMG activity not correlated with muscle stretching. This increase in the variation of EMG activity may be explained by the subject attempting to compensate for the reduced effectiveness of the stretch reflexes but being unable to produce responses coherent with the stimulus.

Procaine block also changed the timing of the reflex responses in that the phase advance of EMG of reflex response ahead of muscle stretch increased by $25^{\circ}-100^{\circ}$ depending on the level of voluntary contraction. This result is consistent with the parallel reflex path hypothesis proposed previously (Neilson, 1972b). It suggests that the total reflex is probably the summation of responses from a number of reflex pathways each having different timing. For example, Ia, Ib, and group II afferent fibres probably each contribute and the timing of the reflex signals in each of these pathways is different, since Ia fibres respond to length and rate of change of length, group II fibres respond to length, and Ib fibres respond to tension and rate of change of tension. A reduction in the magnitude of the reflex signals in Ia and group II fibres caused by the procaine block of gamma efferent fibres, will cause the timing of the total reflex response to approach more closely the timing of the Ib reflex activity alone. A preponderance of inhibitory Ib activity could thus be responsible for the increase in phase lead caused by the procaine infiltration.

Although procaine eliminated tendon reflexes and diminished TSR responses it had no influence at all on the increase in TSR sensitivity which accompanied an increase in voluntary contraction. The slope of TSR gain (ratio of change in IEMG to incremental change in elbow angle) plotted against level of voluntary contraction was unchanged by the gamma efferent block. The gain increased in a manner exactly parallel with the increase before infiltration of procaine. Although it is possible that the gamma efferent fibres were not all blocked and some fusimotor drive may have been present after the procaine block, the similarity of the increase in TSR sensitivity before and after procaine makes it reasonable to conclude that increase in TSR sensitivity with voluntary contraction is not mediated by the gamma efferent fibres.

The failure of gamma efferent block to diminish the control of TSR transmission associated with voluntary contraction suggests that control of reflex sensitivity is not the main function of the fusimotor system. Since alpha and gamma motoneurones are excited in parallel by central stimulation (Matthews, 1964; Koeze, Phillips, and Sheridan, 1968), it may be that movements are produced by setting desired length on the intrafusal fibres as proposed by Merton (1953) and simultaneously signalling anticipated tension requirements to alpha motoneurones. Providing the tension-length characteristics of muscle and load have been correctly anticipated the muscle spindle discharge should remain steady. Any deviation of actual muscle length from desired length would be corrected by reflex action.

Since supraspinal control of TSR sensitivity not mediated by the gamma efferent system th? actual mechanism remains undefined. Three other mechanisms which could possibly be responsible are: (1) direct corticospinal contrô of alpha motoneurone excitability, (2) supras spinal control of pre- and post-synaptic inhibb tion, and (3) control of bias on interneurones $\omega$ associated with reflex arcs. Control of alpha motoneurone excitability cannot explain the increase in phase lead with voluntary contraction which has been described previously (Neilson, 1972c) and has been verified in this study. One might expect the magnitude of the reflex response $\overline{\bar{o}}$ to increase or decrease with alpha motoneurone excitability but one would not expect a change in timing of the reflex signals. Thus control of alpha motoneurone excitability is unlikely to be the sole mechanism causing the increase in TSR? sensitivity. This conclusion is in agreement with Gottlieb, Agarwal, and Stark (1970) who concluded that increased motoneurone excitability due to voluntary activation was unlikely to be 응 responsible for the change in $\mathrm{H}$-reflex sensitivity measured by them during foot pressure tracking because the change in sensitivity preceded and followed a different time course from the change of EMG activity. They proposed that three $N$ distinct signals were concerned with control of $\tilde{\omega}$ 
movement, one to alpha motoneurones, one to gamma motoneurones, and a third to control reflex sensitivity within the spinal cord. Independent control of the sensitivities of separate parallel reflex pathways (say $\mathrm{Ia}, \mathrm{Ib}$, and group II) could change both the magnitude and the timing of the TSR responses. Since both gain and phase characteristics were observed to change with voluntary contraction, independent control of the different parallel pathways by, say, control of background bias on interneurones associated with the reflex arcs, is the mechanism most likely to be responsible for the change in TSR transmission.

The author wishes to thank Associate Professor J. W. Lance and Professor E. P. George for the valuable advice and supervision and the Spastic Centre of New South Wales for financial assistance. The Figures were photographed by the Department of Medical Illustration, University of New South Wales.

\section{REFERENCES}

De Gail, P., Lance, J. W., and Neilson, P. D. (1966). Differential effects on tonic and phasic reflex mechanisms produced by vibration of muscle in man. Journal of Neurology, Neurosurgery, and Psychiatry, 29, 1-11.

Eldred, E., Granit, R., and Merton, P. A. (1953). Supraspinal control of the muscle spindles and its significance. Journal of Physiology, 122, 498-523.

Gassel, M. M., and Diamantopoulos, E. (1964). The effect of procaine nerve block on neuromuscular reflex regulation in man. Brain, 87, 729-742.

Gottlieb, G. L., Agarwal, G. C., and Stark, L. (1970). Interactions between voluntary and postural mechanisms of the human motor system. Journal of Neurophysiology, 33, 365381.
Granit, R., and Henatsch, H. D. (1956). Gamma control of dynamic properties of muscle spindles. Journal of Neurophysiology, 19, 356-366.

Harvey, R. J., and Matthews, P. B. C. (1961). Some effects of stimulation of the muscle nerve on afferent endings of muscle spindles, and the classification of their responses into types A1 and A2. Journal of Physiology, 156, 470-497.

Jansen, J. K. S., and Matthews, P. B. C. (1962). The effects of fusimotor activity on the static responsiveness of primary and secondary endings of muscle spindles in the decerebrate cat. Acta Physiologica Scandinavica, 55, 376-386.

Koeze, T. H., Phillips, C. G., and Sheridan, J. D. (1968). Thresholds of cortical activation of muscle spindles and $\alpha$ motoneurones of the baboon's hand. Journal of Physiology, 195, 419-449.

Lance, J. W. (1970). A Physiological Approach to Clinical Neurology. Butterworths: London.

Landau, W. M., Weaver, R. A., and Hornbein, T. F. (1960). Fusimotor nerve function in man. Differential nerve block studies in normal subjects and in spasticity and rigidity. Archives of Neurology, 3, 10-23.

Matthews, P. B. C. (1964). Muscle spindles and their motor control. Physiological Reviews, 44, 219-288.

Matthews, P. B. C., and Rushworth, G. (1958). The discharge from muscle spindles as an indicator of $\gamma$ efferent paralysis by procaine. Journal of Physiology, 140, 421-426.

Merton, P. A. (1953). Speculations on the servo-control of movement. In The Spinal Cord: a CIBA Foundation Symposium, pp. 247-260. Edited by G. E. W. Wolstenholme. Churchill: London.

Neilson, P. D. (1972a). Speed of response or bandwidth of voluntary system controlling elbow position in intact man. Medical and Biological Engineering, 10, 450-459.

Neilson, P. D. (1972b). Frequency response characteristics of the tonic stretch reflexes of biceps brachii muscle in intact man. Medical and Biological Engineering, 10, 460-472.

Neilson, P. D. (1972c). Interaction between voluntary contraction and tonic stretch reflex transmission in normal and spastic patients. Journal of Neurology, Neurosurgery, and Psychiatry. (In press.)

Rushworth, G. (1960). Spasticity and rigidity: an experimental study and review. Journal of Neurology, Neurosurgery, and Psychiatry, 23, 99-118.

Rushworth, G. (1961). The gamma system in Parkinsonism. International Journal of Neurology, 2, 34-50. 